\title{
Tracking a Moving Objects Using Foreground Detector and Improved Morphological Filter
}

\author{
Diallo Mamadou Saidou \\ School of Telecommunication, Hangzhou Dianzi University, Hangzhou, China \\ Email: dialloms90@yahoo.fr
}

How to cite this paper: Saidou, D.M. (2017) Tracking a Moving Objects Using Foreground Detector and Improved Morphological Filter. Open Access Library Journal, 4: e4152.

https://doi.org/10.4236/oalib.1104152

Received: November 13, 2017

Accepted: December 19, 2017

Published: December 22, 2017

Copyright $\odot 2017$ by author and Open Access Library Inc.

This work is licensed under the Creative Commons Attribution International License (CC BY 4.0).

http://creativecommons.org/licenses/by/4.0/

\begin{abstract}
Mobile object detection is one of the most important steps in computer vision applications such as: medical analysis human-machine interface, robotics, traffic monitoring, and more. In this article, we apply the Gaussian mixing model which is established on the background subtraction. A smoothing method was used for the pre-processing step and a morphological filter was applied to remove unwanted pixels from the background in the other to solve the problem of background noise disturbance. We also demonstrated that filtering foreground segmentation twice with the same morphological structured element but with a different width was used to improve the accuracy of the result. The results show that the proposed method is effective in detecting and tracking moving vehicles, compared to filter segmentation in the foreground only once. Several methods and algorithms have been used to solve this problem. All the methods used before have been effective but also have limits. Some of these methods lose the object when the number of frames is wide while others lose it when it changes direction or rolls at a high speed. In addition, the algorithms proposed for the detection of colors in RGB also lose their objectives when the object changes the color. But the proposed combination in this paper maintains contact with the object without losing it even if it changes direction or speed or the number of frame increases.
\end{abstract}

\section{Subject Areas}

Applications of Communication Systems

\section{Keywords}

Tracking, Background, Foreground, Gaussian Mixture Model, Morphological Filter

\section{Introduction}

For many years in computer vision applications, the detection of the moving 
object is a very important step in the areas of recognition and security. Man by nature can easily detect and distinguish a moving object, but it is difficult for a computer to detect and recognize an object like humans because it does not have the ability to think, which helps detect and recognize moving objects. An object detection system that is not complicated and adaptable is necessary [1]. Moving objects like humans, vehicles, etc. is monitored using a video camera in a visual surveillance scene. The process of extracting the foreground object is an important step in grouping, tracking, and analyzing the activities of the subject object, which is why subtraction of the background plan has been applied in this article [2].

Many related research can be distinguished such as the search by matching [3], search by mean shift [4], search by optical/median flow [5] and bayesian search [6]. Most of the work related to this problem is oriented in terms of the color, size, shape or movement of the mobile, but size plays a very important role in the use of particles. For the extraction of points in the image and the follow-up of these points the algorithm KLT [7] has been much used by many people, it was developed by Kanade and Lucas, it is found in many tasks of tracking of objects. The extraction of points in the first image is done using the Harris detector. To estimate a global motion model in the image, many use the RMRm algorithm developed by J-M Obodez [5]. It allows, from a robust multi-scale estimation, to define a $2 \mathrm{D}$ motion model with several Parameters between two images.

Background subtraction has been chosen as one of the preferred methods for real-time segmentation of moving objects in the alternation of images which is the capture of a still camera [7]. Background subtraction is a process by which the background is cut off from the current frame, i.e. each new frame is subtracted from that pattern and the resultant difference and the binary segmented image represent the exit. The threshold is chosen carefully to reduce the number of false negatives and positives [8] [9]. A false positive is obtained when we choose a small value for the threshold, for the case there will be a lot of foreign pixels. On the other hand, when a high value is chosen, it gives a false negative. In general, we can now summarize the subtraction of the background in: Background initialization, Background modelling, Background maintenance, Foreground detection.

By other, there are many challenges to overcome that include the following: change of lighting variation, movement of background objects, initialization of shadows with moving objects and complex dynamic backgrounds such as swaying of trees, ripples in water.

The use of Background Subtraction Methods (BS) could find a way to deal with these problems [10] which is why BS is adopted in this article. The sound of the video or image can make the detection of objects in motion very complicated, which can make detection difficult. Different ways of eliminating noise have been developed by many researchers. In this article we present a modified morphological filtering process. The method we have proposed re- 
duces the effects of the noise of the detected object, which improves the efficiency of the detection process. This article has described a task that has been successful in achieving satisfactory results in detecting and tracking the moving object in the video. The task is to keep an object selected by the user in the center of the image whatever its movements. This algorithm allows good robustness to the very strong disturbances due to video transmission and operates at a bit rate close to the video bit rate. Experimentation in real conditions shows the effectiveness of the proposed system. This document is organized in the following order. Section II presents a brief presentation of our proposed method. Section III presents the results of the simulation and Section IV presents the conclusion.

\section{Proposed Method}

The method is structured in five steps which are indicated in the figure below (Figure 1).

The input video smoothes the process where the noise is reduced and the image is blurred. Second, the background subtraction based on the Gaussian mixing model is used to detect the moving object. Third, by using thresholding, the binary image is obtained. Fourth, morphological filtering is applied twice, which has had a considerable effect on noise reduction and merges the separate blobs associated with an object. Fifth, the connected blobs are tagged to the individual object by the bounding box. Etc.

1) Imput video (smoothing):

The smoothing process is used to create a fuzzy image and reduce the noise of

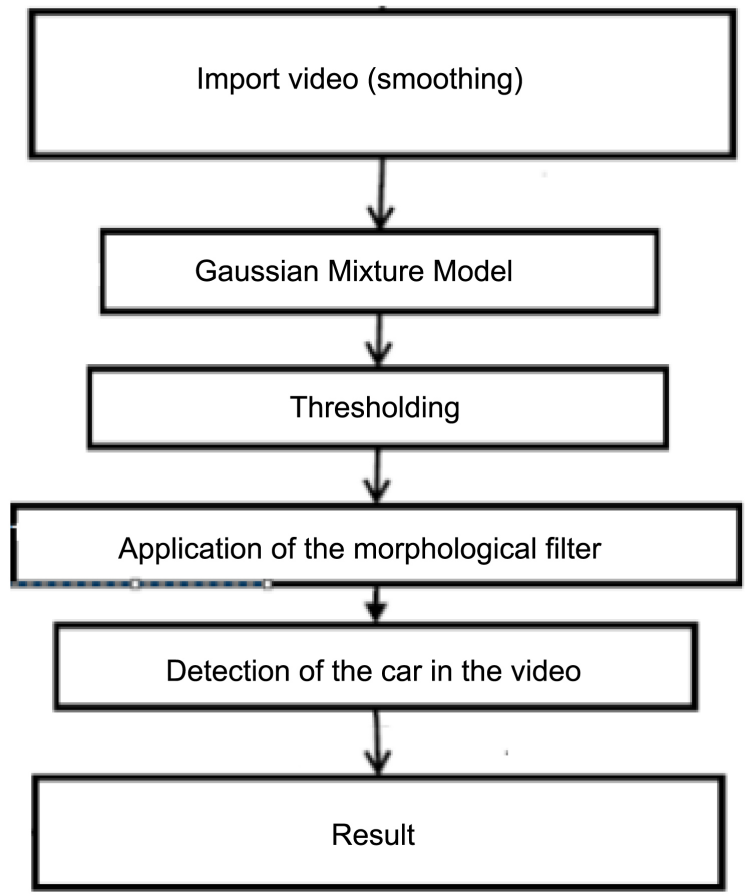

Figure 1. Diagram of the proposed method. 
an image. This is a method very commonly used in image processing; it is mainly used in pre-treatment for background subtraction that depends on GMM [11]. In this article, we will use $5 \times 5$ average box filters, because of the real-time processing. The cost of calculation is reduced, unlike others, for example the Gaussian filter [12].

2) Gaussian Mixture Model (GMM)

GMM represents a combination of $n$ Gaussian distribution that corresponds to the distribution of the pixel strength in the current frame. The probability of intensity in the frame at time $\mathrm{t}$ can be modeled as follows:

$$
p\left(x_{t}\right)=\sum_{n=1}^{n} w_{n, t} x N\left(x_{t}, \mu_{n, t}, \sum_{n, t}\right)
$$

where, $w_{n, t}, \mu_{n, t}$ and $\sum_{n, t}$ are respectively the weight estimation, mean, and the covariance matrix of Gaussian $n^{\text {th }}$, the variance of the Gaussian $n^{\text {th }}$ matrix is referred from standard deviation

$$
\sum_{n, t}=\mu_{n, t} x_{t}
$$

The intensity of the pixel $x_{t}$ is evaluated with respect to each of the Gaussian elements at the nearest distribution, the parameters, $w_{n, t}, \mu_{n, t}$ and, $\sum_{n, t}$ are updated. The set of background pixels is adaptably identified. With this, it is more preferable to use GMM to remove all potential background pixels. GMM established on the background subtraction is resistant against any change of lighting [13].

3) Morphological filtering

Background subtraction, using GMM, the output contains a lot of noise and the moving object separates, the two fundamental operations of the morphological filtering are erosion and dilation [14]. In this article we will use the aperture filter, which has been successfully applied to the binary image. Morphological filtering is a process that is mainly used to reduce noise in detected images. A morphological operation gives the best edges to moving vehicles and produces the best performance. Some unwanted noises are part of in the foreground segmentation process, which makes it not perfect. We decided to double-filter foreground segmentation to remove the noise [15]. Using the blob vision analysis object, this one found a link component of the bounding box corresponding to the moving car. Any blob of less than 150 pixels is not accepted.

\section{Results of the Simulations}

The video used in this work is that of an automobile in motion along a trajectory, this video is in AVI format (length 60 seconds, frame width 320, frame height 240, data rate $200 \mathrm{kbps}$, total bitrate $200 \mathrm{kbps}$, frame rate 25 frame/second) (Figure 2).

Using GMM, Background subtraction, we can notice that the output contain a lot of noise and the detection of the moving object becomes troubled (Figure 3). To remedy this problem, we need to apply the morphological operations to denoise the image. 


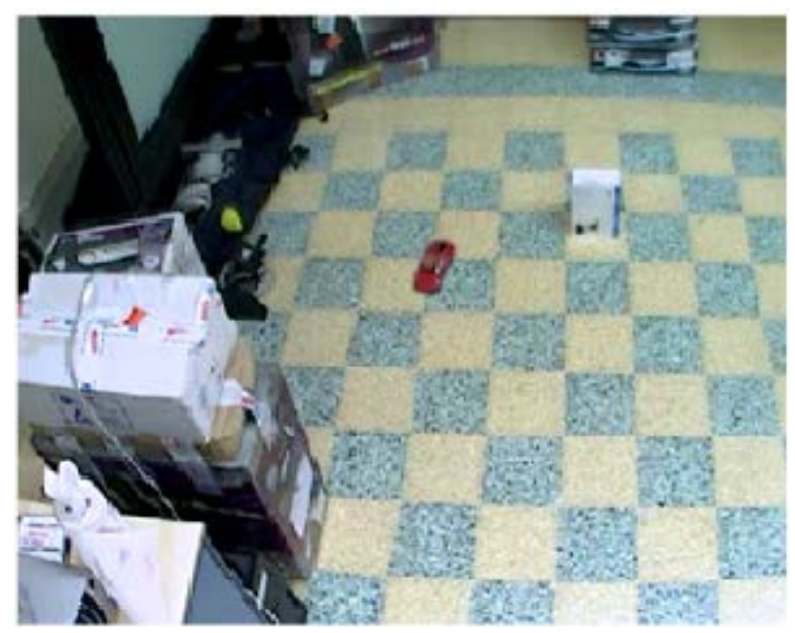

Figure 2. The original video.

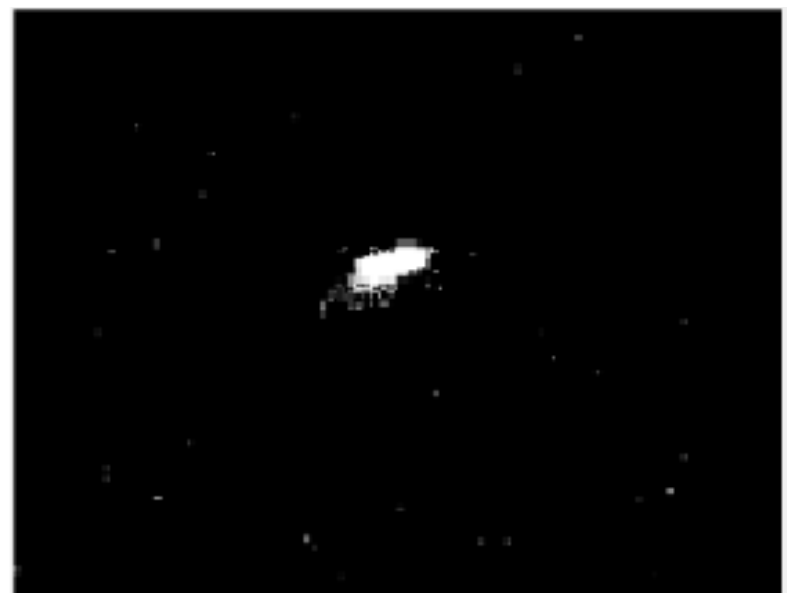

Figure 3. Background image with Gaussian additive noise.

Morphological filtering is used to reduce noise in image detection, by applying that technic, we can see on (Figure 4) that all the noises have been removed by the morphological filter. And by that, we obtain a clean foreground. On this image we can see that all the noises have been removed by the morphological filters. This saw us that morphological operation gives the best assets for moving vehicles and produces the best performance.

The Figure 5 shows the result of the algorithm.

\section{Conclusion}

The aim of the search is to propose an algorithm allowing to follow an object in motion in a video. In this paper we have shown that the background substraction method detects and tracks the trajectory of a car moving in a video without losing it. Based on all these results, we can conclude that, the background subtraction method gives us a better and more accurate result for the detection of moving vehicles. It should be noted that filtering the segmentation of the foreground several times makes it possible to obtain better segmentation for 


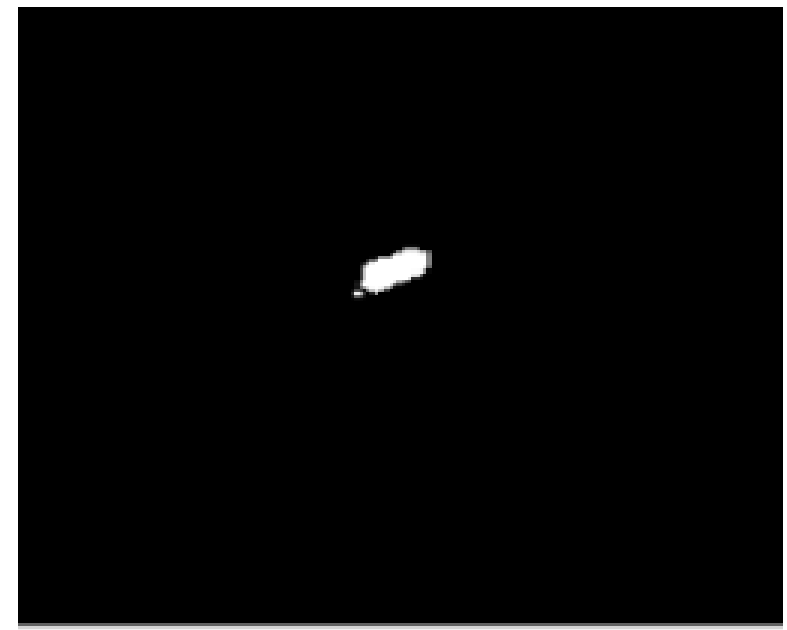

Figure 4. The Gaussian Noise is cleaned by the Morphological filtering.

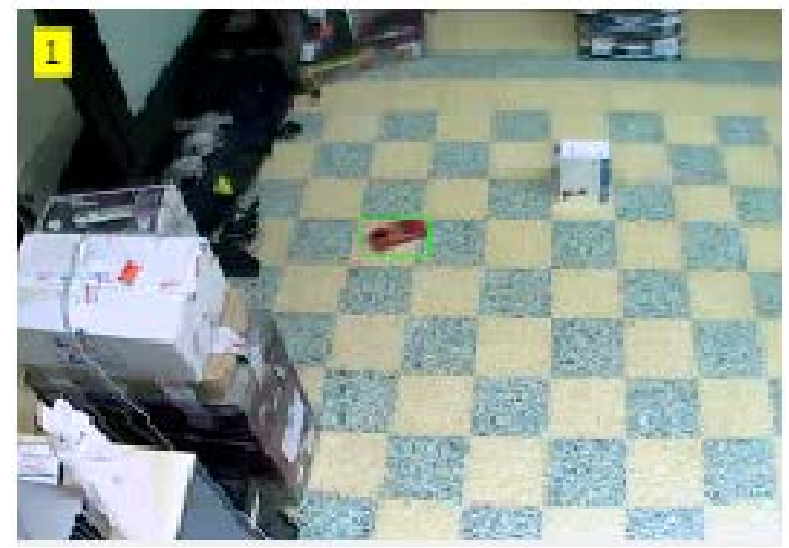

Figure 5. The car is detected buy the algorithm.

moving vehicles and produces a good performance due to the elimination of noise which now makes the blob analysis to produce a precise delineation to each of the vehicles. The result of the simulation shows the effectiveness of the proposed method.

\section{Discussions}

Despite the fact that the results obtained are satisfactory, this work can be improved for more performance. In the future, it will be interesting to make projects, to resume the work done and to complete it. So that it can first improve its speed of execution by simplifying it, improving its result by improving the techniques, or by facilitating its use. For this, it would be possible to study new algorithms that could improve the results of a type of video or object.

\section{References}

[1] Khurana, K. and Awasthi, R. (2013) Techniques for Recognizing Objects in Image and Object Detection. International Journal of Advanced Research in Computer 
science Engineering and Technology, 2, 1385-1387.

[2] Crane, H. and Steele, C. (1968) Translation-Tolerant Mask Matching Using Non coherent Reflective Optics. Recognition, 1, 129-136.

[3] Han, B., Joo, S.-W. and Davis, L.S. (2007) Probabilistic Fusion Tracking Using Mixture Kernel-Based Bayesian Filtering. In IEEE 11 th International Conference on Computer Vision, Rio de Janeiro, Brazil, September 2007, 14-20.

[4] Klein, J., Lecomte, C. and Miche, P. (2007) Fast Color-Texture Discrimination: Application to Car-Tracking. In IEEE ITSC, Seattle, WA, USA, 30 Sept.-3 Oct. 2007, 546-541. https://doi.org/10.1109/ITSC.2007.4357765

[5] Bouwmans, T., Baf, F. and Vachon, B. (2008) Background Modelling Using Mixture of Gaussians for Foreground Detection-A Survey. Recent Patents on Computer Science, 1, 219-237. https://doi.org/10.2174/2213275910801030219

[6] Khurana, K. and Awasthi, R. (2013) Techniques for Object Recognition in Images and Multi-Object Detection. International Journal of Advance Research in Computer Engineering and Technology, 2, 1383-1387.

[7] Dimitrijevic, M. (2006) Detecting Human Body Posture Using Spatial-Temporal Bayesian Models. International Conference on Intelligent and Advanced Systems, 10, 127-139.

[8] Vahora, S., Chauhan, N. and Prajapati, N. (2012) A Robust Method for the Detection of Moving Objects Using the Modified Statistical Method. International Journal of Advanced Information Technology (IJAIT), 2, 204-209.

https://doi.org/10.5121/ijait.2012.2106

[9] Bobick, A. and Davis, J. (2001) The Recognition of Human Movements Using Temporal Models. IEEE Transactions on Model Analysis and Artificial Intelligence, 23, 257-267.

[10] Haritaoglu, I., Harwood, D. and Davis, L.S. (2000) W4: Real-Time Monitoring of the Activities of People. IEEE Transactions on Artificial Intelligence and Model Analysis, 22, 809-830.

[11] Azarbayejani, T.D. and Pentl, P. (1997) Real-time Monitoring of the People. IEEE Transactions on Artificial Intelligence and Model Analysis, 19, 780-785.

[12] Stauffer, C. and Grimson, W. (1999) Adaptive Background Mixture Models for Real-Time Monitoring in Computer Vision and Pattern Recognition. IEEE Computer Society, 2, 2246-2252.

[13] Stauffer, C., Eric, W. and Grimson, L. (2000) Activity Learning Models Using Real-Time Monitoring. IEEE Transactions on Model Analysis and Artificial Intelligence, 22, 747-757.

[14] Bouwmans, T., Baf, F. and Vachon, B. (2008) Background Modeling Using a Gaussian Mix for Leading Detection-An Investigation. Recent Patents on Computer Science, 1, 219-237. https://doi.org/10.2174/2213275910801030219

[15] Schick, A., Bauml and Stiefelhagen, R. (2012) Improvement of Foreground Segmentations with Random Fields of Probabilistic Markov Super Pixels. Computer Vision and Pattern Recognition (CVPR), 2012 IEEE Computer Society Conference on IEEE, 27-31. 